\title{
Dynamic threshold-based congestion control in wireless multimedia networks
}

\author{
Cuthbert Abigaba Mirembe ${ }^{1}$, Roseline Nyongarwizi Akol ${ }^{2}$, Julius Butime ${ }^{2}$ \\ ${ }^{1}$ Faculty of Engineering, Department of Electrical \& Electronic Engineering, Kyambogo University, P. O. Box 1 Kyambogo, Uganda \\ ${ }^{2}$ Department of Electrical \& Computer Engineering, School of Engineering, Makerere University, P. O. Box 7062 Kampala, Uganda \\ *Corresponding author E-mail: cmabigaba@yahoo.com
}

\begin{abstract}
The last few decades have seen a tremendous growth of wireless networks demands due to the need to meet different multimedia applications available over the network. The demand has resulted into greater competition for the scarce available network resources and has introduced congestion in such networks that adversely affect the quality of service requirements of the applications. Call admission control is a key element if provision of quality of service in wireless networks is to be guaranteed. In this paper, a call admission control policy for wireless communication networks based on the use of dynamic threshold-based channel allocation scheme is proposed. The scheme deals with two traffic classes; voice and data with two different but equal channel thresholds, one for each traffic type assumed. The effect of the control parameters on the quality of service parameters is illustrated and analyzed.
\end{abstract}

Keywords: Buffer, Call Blocking Probability, Congestion, Handoff, QoS

\section{Introduction}

Mobile wireless networks have experienced tremendous growth in the recent past, and this growth trend is likely to continue in future. Apart from an increase in the number of users, more demanding applications have emerged, resulting in more resource requirements. These services require a backbone network that can support connections with guaranteed quality of service $(\mathrm{QoS})$ requirements. The wireless channel must not reach the congestion point, as this would lead to increased call drops and delays, a situation that is grossly unfair to calls through such a network.

Call admission control (CAC) and network resource allocation are some of the design issues in wireless networks. CAC schemes determine whether to accept or reject new calls with due consideration of the available network resources maintaining the QoS and ensuring the existing calls are not affected [1,2]. Network resource allocation ensures incoming connection requests are accepted to avoid congestion [3]. The main QoS parameters in a cellular concept are the new call blocking and handoff call blocking probabilities [4] as well as the data call delay due to buffering.

A mobile user trying to communicate with another user must first obtain a channel from the base station. Whenever a channel is available, it is granted to the user and the call is accepted otherwise the call is blocked. The channel is freed either when the call is completed or when the user moves to the next cell before completing the call. The latter is called handoff and while performing handoff, the mobile unit demands that the base station in the cell that it moves into allocates it a channel or else the handoff call is blocked [4]. Blocking such a call is annoying to the user; instead a buffer is introduced to store the call until a channel is available to transmit it. The buffered call experiences delay for the duration of the handoff call.

To improve the performance of the cellular networks, channel allocation is usually done statically though some dynamic channel allocation mechanisms [5, 6]. Dynamic threshold and buffer management at various locations in the network drastically reduce congestion problems; since these determine the rate at which traffic enters the network. In this paper, a dynamic threshold-based guard channel policy is proposed to handle both the new and handoff voice and data calls with buffers at various locations in the network to handle the handoff call rather than getting blocked. The policy deals with distinct types of traffic types, voice and data but can be extended to multiple traffic classes. 
This paper is organized in sections as follows: Section 2 describes the call admission policy proposed, Section 3 analyzes the mathematical model for the proposed policy and results are presented in Section 4 while Section 5 is the conclusion.

\section{Proposed Call Admission Control Policy}

A single cell in isolation within a cellular system that deals with two types of traffic: voice and data was considered. It is assumed that the call processing entities of the system (the Base Station, Base Station Controller or Mobile Switching Center) are capable of identifying the call type at any moment. The available resources are the number of channels in the cell and the buffer capacity to queue handoff calls in case no channels are available. Since wireless resources are limited, it is assumed that the number of channels is constant. The buffer capacity can be changed depending on the required call blocking probabilities; an increase in buffer capacity increases the overall channel capacity.

The proposed admission policy deals with two categories of parameters. The QoS parameters, which comprise the voice call blocking probability for new and handoff calls, $P_{N v}$ and $P_{H v}$ respectively; the data call blocking probability for new and handoff calls, $P_{N d}$ and $P_{H d}$ respectively; and the delay experienced by handoff data calls due to buffering $D_{H d}$. The others are CAC policy parameters which include the buffer size $B$ which determines the value of the buffer size to queue the handoff data calls; the new threshold $T_{N d}, T_{N v}$ that determines the total number of the channels allocated to data and voice calls after which the new data calls are blocked.

The conditions to accept or reject a call for the call admission control policy proposed employing the above methods are illustrated in flow chart in Fig. 1.

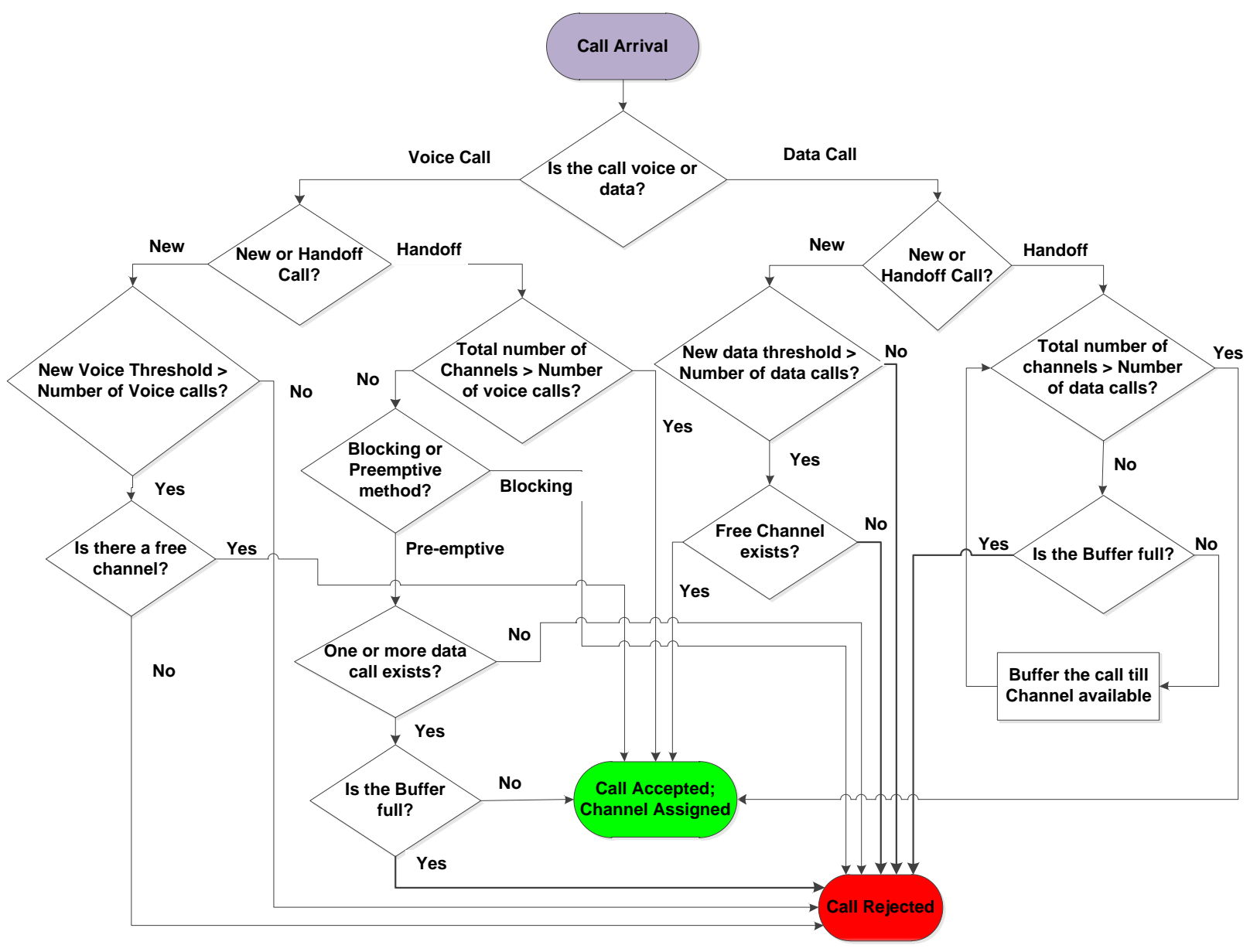

Fig. 1: Call Admission Flow Chat 


\section{Mathematical Model Analysis}

The single cell model parameters assumed are the total number of channels $C$, a call request buffering capacity $B$ and channel holding time $\mu$ equal for voice and data calls. Since each of the two traffic types, voice and data can either be new or handoff, there are four different arrival rates: $\vartheta_{N v}$ for new voice calls; $\vartheta_{N d}$ for new data calls; $\vartheta_{H v}$ for handoff voice calls; and $\vartheta_{H d}$ for handoff data calls. For each call type, the total arrival rate is [7]:

$$
\begin{gathered}
\vartheta_{T v}=\vartheta_{N v}+\vartheta_{H v} \\
\vartheta_{T d}=\vartheta_{N d}+\vartheta_{H d}
\end{gathered}
$$

As only two input traffic types have been considered, the system mathematical model is based on a 2-dimensional Markovian chain in which each state represents the number of voice and data calls in such a system. The total number of calls at each state is $n_{\text {Total }}$, where

$$
n_{\text {Total }}=n_{v}+n_{d}
$$

with number of voice calls at a certain state, $n_{v}$, ranging from 0 to $C$ and number of data calls at a certain state, $n_{d}$, ranging from 0 to $C+B$.

A standard procedure was followed to derive the state probabilities [8, 9]. The steady-state equations for the state probabilities were written in terms of the state transition rate matrix $Q$ and the state probability vector $\Pi$ whose product results into a zero matrix while the elements of state probability vector add up to one, that is,

$$
\Pi . Q=0
$$

and

$$
\sum \pi_{i}=1
$$

where

$$
\Pi=\left[\begin{array}{llllll}
\pi_{0,0} & \pi_{0,1} & \pi_{0,2} & \ldots \ldots . & \pi_{n_{v}, n_{d}} & \ldots . . .
\end{array}\right]
$$

and

$$
Q^{T}=\left[\begin{array}{cccccccc}
-\left(\vartheta_{T v}+\vartheta_{T d}\right) & \mu & 0 \ldots & 0 & \mu & 0 & \ldots . . & 0 \\
\vartheta_{T d} & -\left(\vartheta_{T v}+\vartheta_{T d}+\mu\right) & 2 \mu & 0 \ldots . & 0 & 2 \mu & 0 \ldots & 0 \\
0 & \vartheta_{T d} & -\left(\vartheta_{T v}+\vartheta_{T d}+2 \mu\right) & 3 \mu & \ldots . & 0 & 3 \mu & 0 \\
. & . & . & . & & & & . \\
. & . & . & . & & & & .
\end{array}\right]
$$

A MATLAB program was written to generate and solve (4) to obtain the state probability vector. The state probabilities, the values of the QoS parameters were calculated [7] using (6) to (11).

The new data call blocking probability $P_{N d}$ is given by

$$
P_{N d}=\left(\sum_{n_{v}=0}^{C} \sum_{n_{d}=T_{N d}}^{C+B-n_{v}}\left(\pi_{n_{v}, n_{d}}\right)\right) \frac{\vartheta_{N d}}{\vartheta_{T d}+\vartheta_{T v}}
$$

The new voice call blocking probability $P_{N v}$ is given by

$$
P_{N v}=\left(\sum_{n_{v}=T_{N v}}^{C} \sum_{n_{d}=0}^{C+B-n_{v}}\left(\pi_{n_{v}, n_{d}}\right)\right) \frac{\vartheta_{N v}}{\vartheta_{T d}+\vartheta_{T v}}
$$

The handoff data call blocking probability, $P_{H d}$ is given by

$$
P_{H d}=\left(\sum_{n_{v}=0}^{C} \pi_{n_{v}, C+B-n_{v}}\right) \frac{\vartheta_{H d}}{\vartheta_{T d}+\vartheta_{T v}}
$$


The handoff voice call blocking probability $P_{H v}$ is given by

$$
P_{H v}=\left(\sum_{n_{v}=0}^{C} \sum_{n_{d}=C-n_{v}}^{C+B-n_{v}}\left(\pi_{n_{v}, n_{d}}\right)\right) \frac{\vartheta_{H v}}{\vartheta_{T d}+\vartheta_{T v}}
$$

using the blocking method and

$$
P_{H v}=\left(\sum_{n_{v}=0}^{C} \pi_{n_{v}, C+B-n_{v}}\right) \frac{\vartheta_{H v}}{\vartheta_{T d}+\vartheta_{T v}}
$$

using the pre-emptive method.

The average data handoff call delay time $D_{H d}$ is given by

$$
D_{H d}=\frac{\sum_{n_{v}=0}^{C} \sum_{n_{d}=C-n_{n}}^{C+B-n_{v}}\left(\pi_{n_{v}, n_{d}}\right)\left\{n_{v}+n_{d}\right\}}{\vartheta_{H d}\left(1-P_{H d}\right)}
$$

\section{Presentation of results}

The effect of the threshold values and the buffer size on new voice/data call blocking probability $\left(P_{N v} / P_{N d}\right)$, handoff voice/data call blocking probability $\left(P_{H v} / P_{H d}\right)$ and delay due to data call buffering $\left(D_{H d}\right)$ is investigated using (6) to (11) where the policy parameters assumed are: total number of channels, $C=25$; two values of the average channel holding time, $\mu, 7$ and 15 seconds; an arrival rate voice and data calls, $\vartheta$, between 0.2 and 0.4 Erlang/channel and buffer capacity, $B$ of 0 to 5 channels. In all simulations the new data threshold $\left(T_{N d}\right)$ is never zero.

Fig. 2 illustrates the effect of varying the new voice threshold $\left(T_{N v}\right)$ on new voice call blocking probability ( $\left.P_{N v}\right)$, for different values of channel holding time, call arrival rate and buffer size. It is clear that there is a considerable effect of $T_{N v}$ on $P_{N v}$ especially for lower values of the threshold, where we observe a sharp decrease in $P_{N v}$.

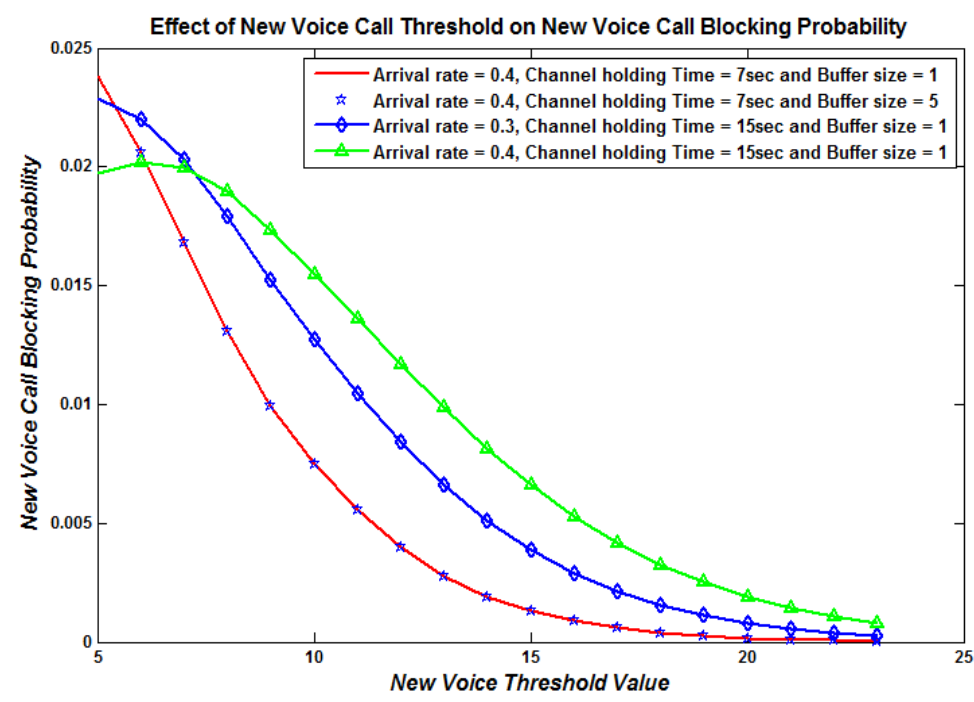

Fig.2: Effect of New Voice Threshold on New Voice Call Blocking Probability

Furthermore, it can be observed that $P_{N v}$ tends to be constant for the values of $T_{N v}$ approaching the number of available channels $(C=25)$. It is seen that for a given value of $T_{N v}$, the blocking probability $P_{N v}$ is generally high for higher arrival rates especially for higher $T_{N v}$ values in agreement with [10]. It is also evident that $P_{N v}$ varies inversely with the 
channel holding time for lower values of $T_{N v}$ but the two are proportional for higher values of $T_{N v}$ approaching $C$. Lastly, for a given arrival rate and channel holding time buffering has no effect on $P_{N v}$ for all $T_{N v}$.

The effect of varying the new voice threshold $\left(T_{N v}\right)$ on new data call blocking probability $\left(P_{N d}\right)$ is shown in Fig. 3 . It is evident that varying $T_{N v}$ has no effect on $P_{N d}$ for a given call arrival rate, channel holding time and buffer size. For a given call arrival rate $P_{N d}$ decreases as channel holding time increases. It can further be seen that when the call arrival rate increases $P_{N d}$ decreases for a given channel holding time. The introduction of buffer capacity slightly increases the new data call blocking probability, other parameters kept constant.

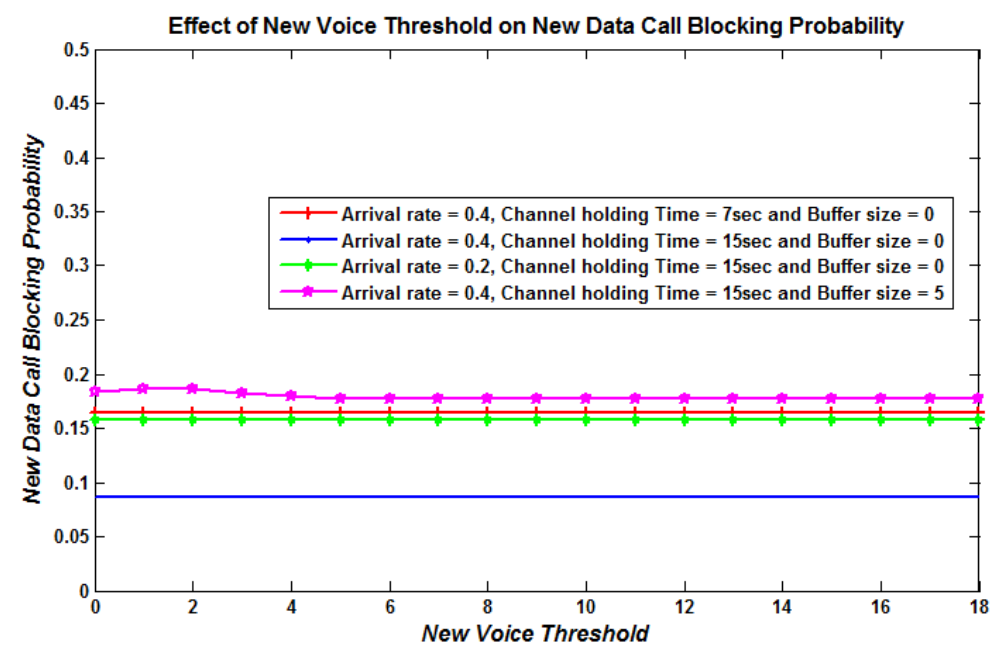

Fig. 3: Effect of New Voice Threshold on New Data Call Blocking Probability

Fig. 4 shows the effect of new voice threshold $\left(T_{N v}\right)$ on handoff voice call blocking probability $\left(P_{H v}\right)$. Generally, $P_{H v}$ increases with $T_{N v}$ and saturates to a maximum of $P_{H v} \approx 0.5$ as $T_{N v}$ approaches $C$. In Fig. 4 it is clear that for a given $T_{N v}, P_{H v}$ increases when the arrival rate drops while $P_{H v}$ is seen to decrease with increase in buffer size in agreement with findings in [11]. On the other hand $P_{H v}$ is observed to decrease as the channel holding time is increased.

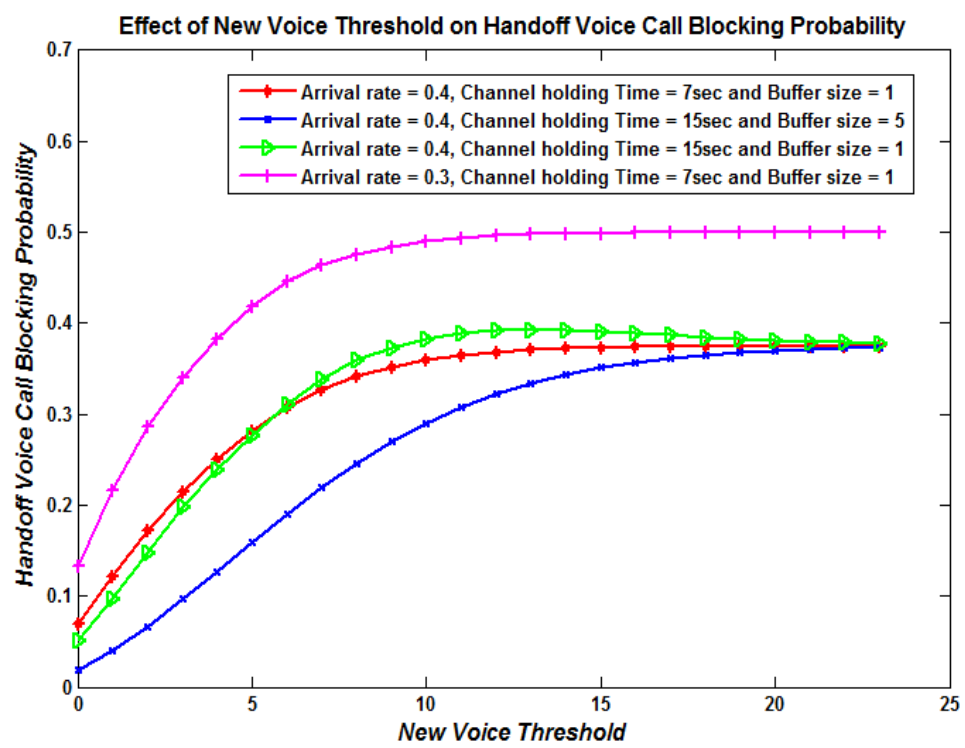

Fig. 4: Effect of New Voice Threshold on Handoff Voice Call Blocking Probability 
Fig. 5 is an illustration of the variation of handoff data call blocking probability ( $P_{H d}$ ) for different new voice threshold values $\left(T_{N v}\right)$, arrival rates and buffer sizes. There is a general increase in $P_{H d}$ as $T_{N v}$ increases which saturates as the threshold value approaches the channel capacity $(C=25)$. It is observed that a change in arrival rate while other parameters are held constant does not affect $P_{H d}$; whereas increasing the buffer size and channel holding time is observed to significantly lower $P_{H d}$ as in [11]. However, this improvement is at the expense of increasing the average delay time added to the call connection time for handoff data calls as in Fig. 6.

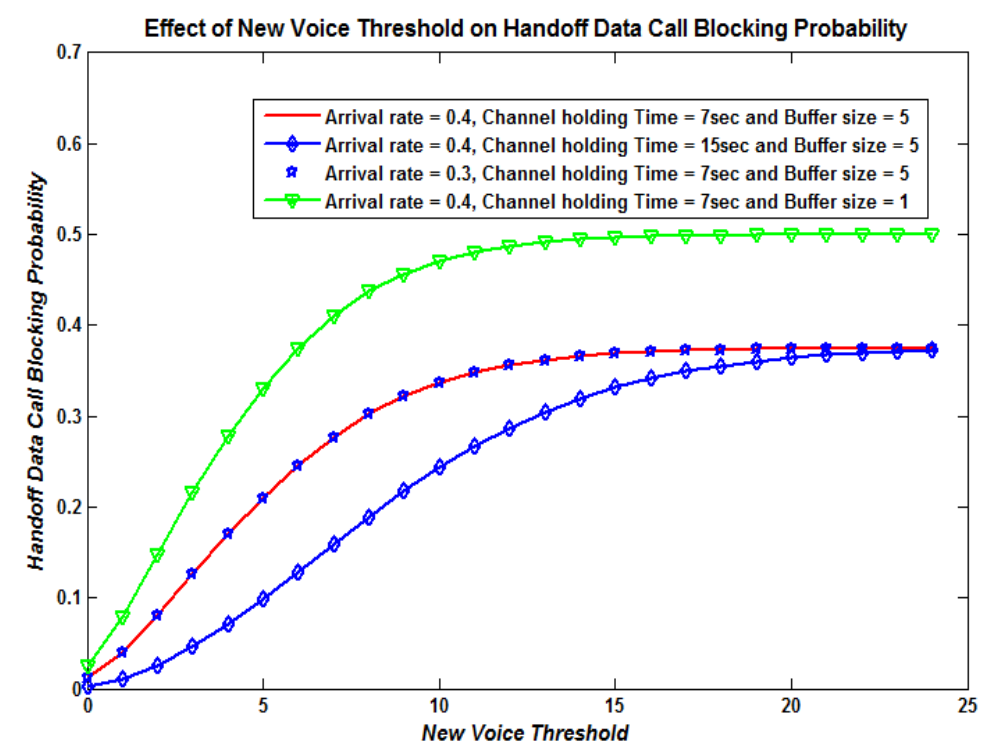

Fig.5: Effect of New Voice Threshold on Handoff Data Call Blocking Probability

There is a general increase in $P_{H d}$ as $T_{N v}$ increases which saturates as the threshold value approaches the channel capacity $(C=25)$. It is observed that a change in arrival rate while other parameters are held constant does not affect $P_{H d}$; whereas increasing the buffer size and channel holding time is observed to significantly lower $P_{H d}$ as in [11]. However, this improvement is at the expense of increasing the average delay time added to the call connection time for handoff data calls as in Fig. 6.

In Figure 6 the effect of new voice threshold $\left(T_{N v}\right)$ on handoff data call delay $\left(D_{H d}\right)$ illustrated. The delay is seen to increase when the buffer is increased; the delay also clearly increases as the arrival rate increases. It is evident that $D_{H d}$ depends on the new voice threshold, the channel holding time and buffer size; an increase in any of these parameters increases the handoff data call delay.

Finally the combined effect of the new voice and new data thresholds was analyzed for fixed values of call arrival rate, channel holding time and buffer size. Fig. 7 shows the combined effect on the new voice call blocking probability for a total arrival rate (new and handoff) of $\vartheta=0.4$, buffer size $B=5$ and channel holding time of 7 seconds.

The variation of both thresholds is observed to impact on the new voice call blocking probability which in each case diminishes as the new data threshold value approaches the channel capacity $(C=25)$. Minimum values of $P_{N v}$ are achieved for any $T_{N d}$ value as long as $T_{N v}$ is close to the channel capacity and vice versa. It is further observed that maximum $P_{N v}$ occurs at low $T_{N v}$ values. 


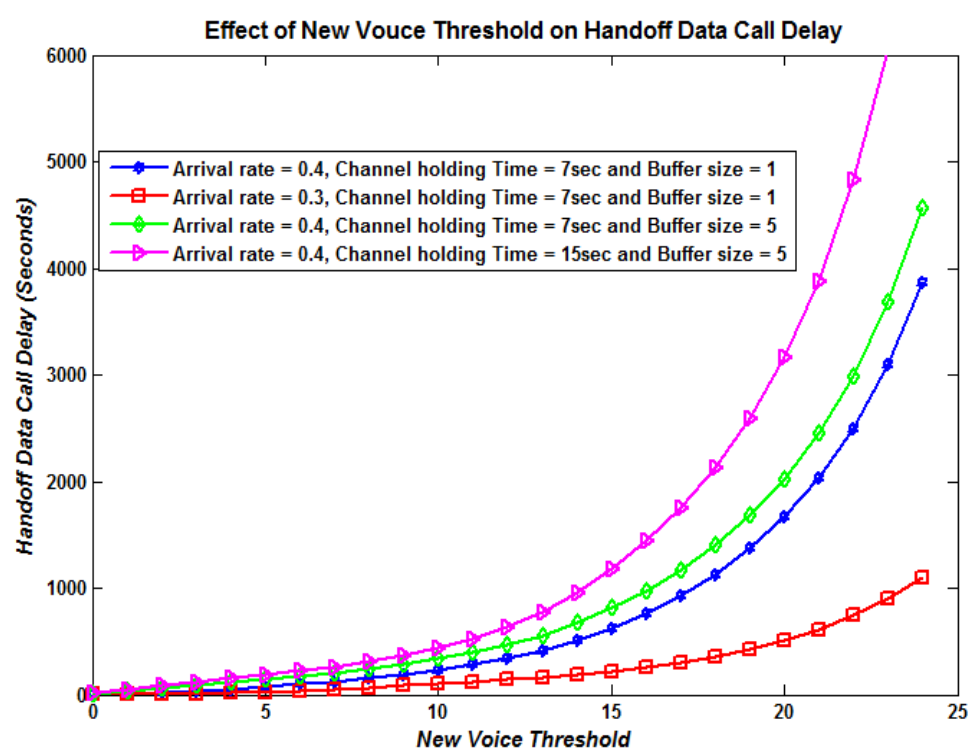

Fig. 6: Effect of New Voice Threshold on Handoff Data Call Delay due to Buffering

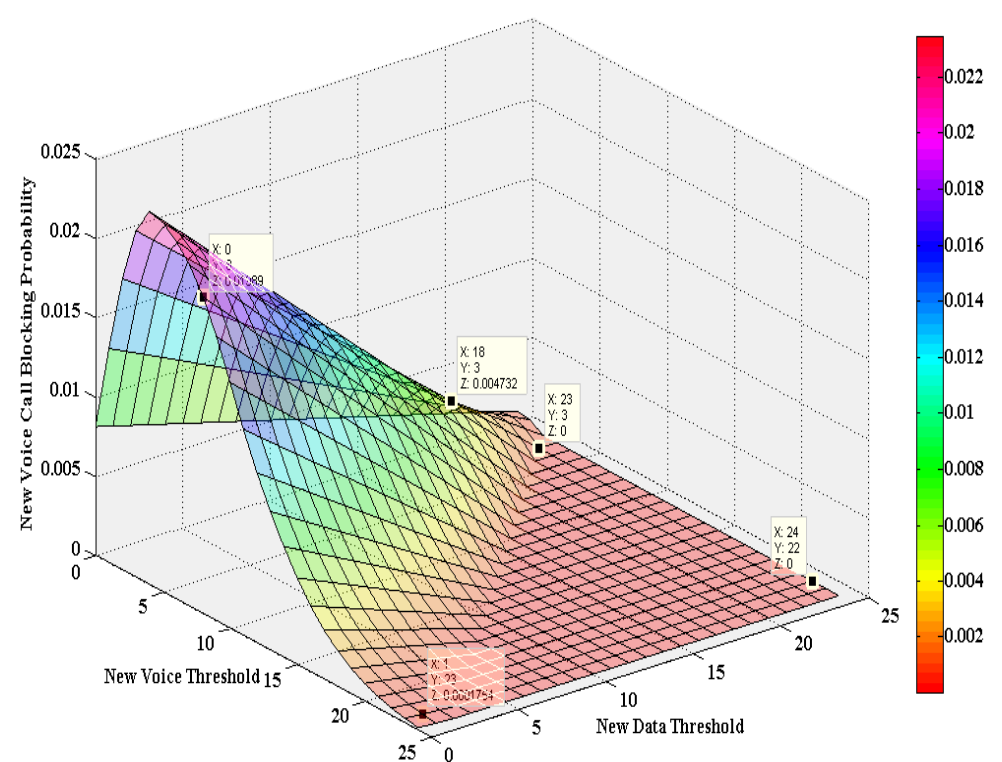

Fig. 7: Combined Effect of New Voice/Data Thresholds on New Voice Call Blocking Probability

In Fig. 8, the combined effect of the new voice threshold $\left(T_{N v}\right)$ and new data threshold $\left(T_{N d}\right)$ on the new data call blocking probability ( $P_{N d}$ ) is illustrated. For $T_{N d} \leq 10, P_{N d}$ saturates to a value of $P_{N d} \approx 0.06$ for all values of $T_{N v}$. $T_{N d}$ is observed to mainly impact on $P_{N d}$ when $T_{N v} \leq 15$. In the regions $T_{N d} \geq 10$ and $T_{N v} \leq 15, P_{N d}$ goes to a symmetrical minimum that drops as $T_{N v}$ decreases to zero and $T_{N d}$ increases towards the maximum channel capacity.

In Fig. 9 the effect of changing values of the new voice threshold and new data threshold on the handoff voice call blocking probability $\left(P_{H v}\right)$ is illustrated. $P_{H v}$ is observed to vary symmetrically for new voice and new data threshold values and saturating threshold values above 15 to $P_{H v} \approx 0.25$. Minimum $P_{H v}$ occurs when both thresholds are close zero. 


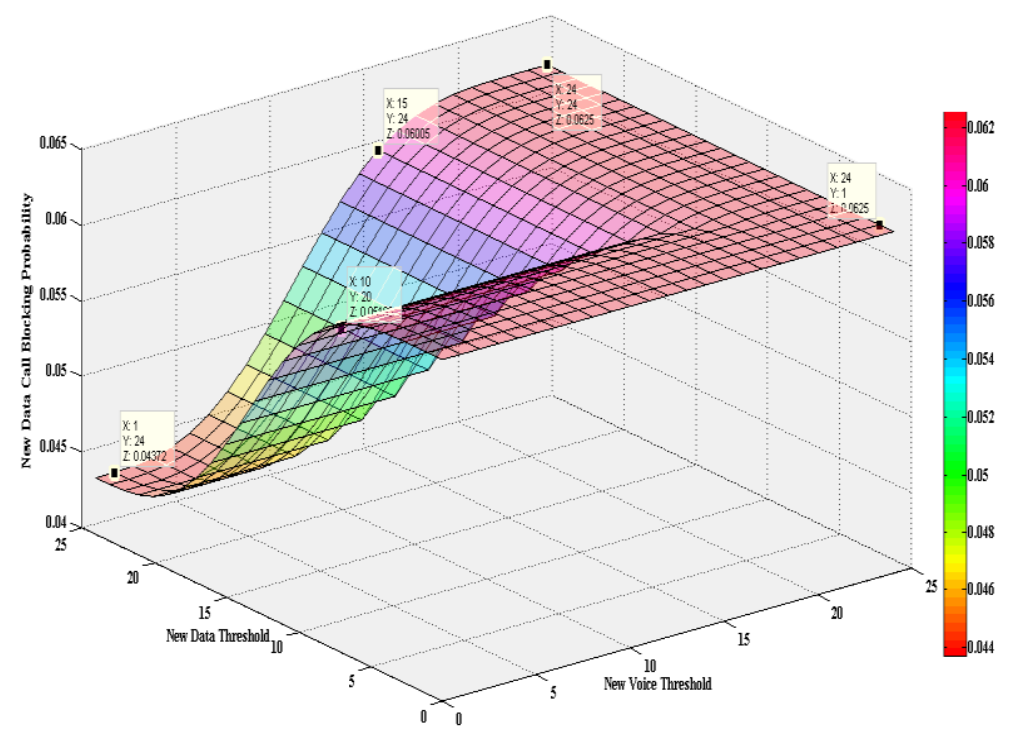

Fig. 8: Combined Effect of New Voice/Data Thresholds on New Data Call Blocking Probability

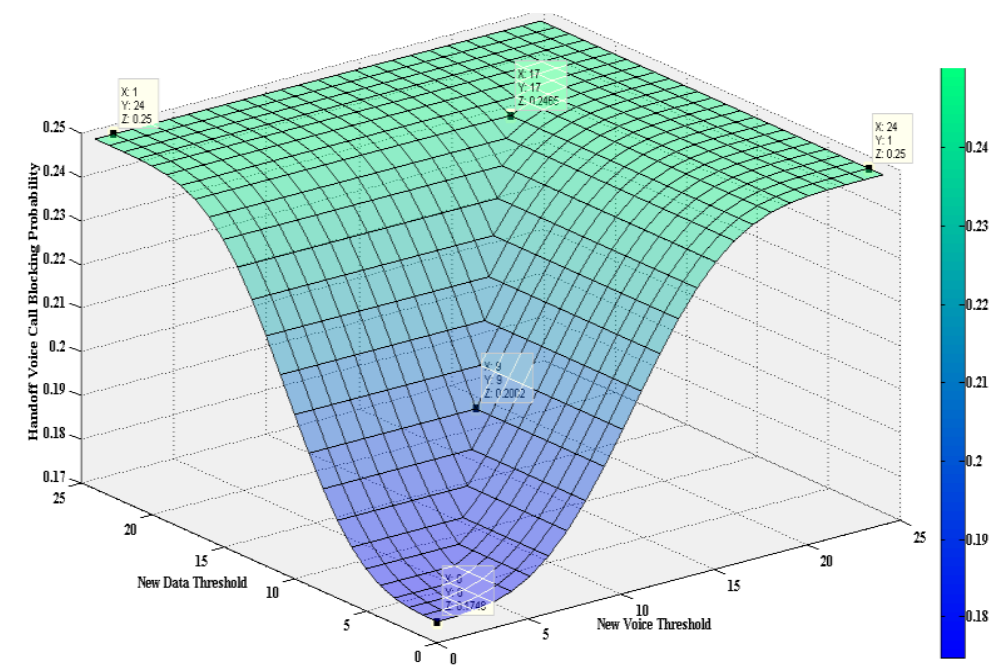

Fig. 9: Combined Effect of New Voice/Data Thresholds on Handoff Voice Call Blocking Probability

In Fig. 10 the effect of changing values of the new voice threshold $\left(T_{N v}\right)$ and new data threshold $\left(T_{N d}\right)$ on the handoff data call blocking probability $\left(P_{H d}\right.$ ) is illustrated. Maximum $P_{H d}$ is observed when $T_{N d} \geq 10$ and $T_{N v} \leq 10$; else where the probability diminishes to a minimum especially as $T_{N v}$ approaches the channel capacity.

\section{Conclusion}

In this paper, a call admission policy for wireless multimedia networks based on modifying the guard channel method and introducing a buffer for queuing handoff data calls has been presented. The effect of new call threshold, call arrival rate, channel holding time and buffering on QoS parameters for voice and data has been analyzed. All QoS parameters increase with the threshold values except the new voice call blocking probability (decreases) and the new data call blocking probability (independent). Except handoff data call blocking probability where no difference was observed, all the other QoS parameters increase with increase in call arrival rate. Similarly all parameters increase with an increase in buffer size except the new voice call blocking probability for which buffer size variation shows no effect.

In all cases, variation of new data and voice thresholds is observed to significantly impact on all QoS parameters except the handoff data call delay for which variation of the new data threshold for a given new voice threshold value has no impact. This is manifested in the combined effect of the new voice and data threshold on QoS parameters analysis. 


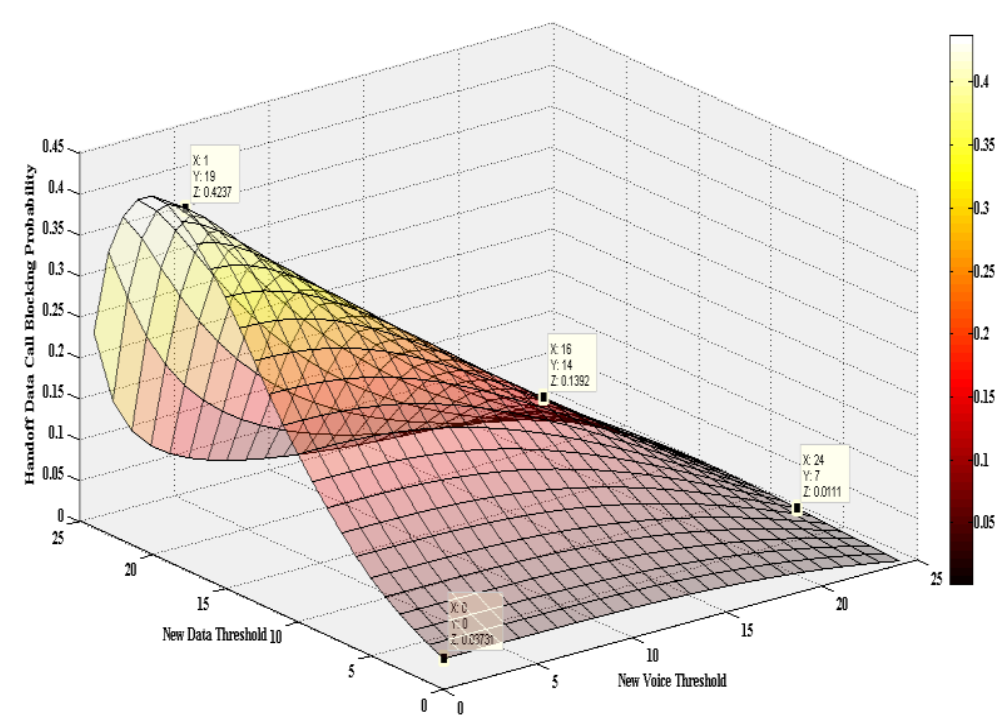

Fig. 10: Combined Effect of New Voice/Data Thresholds on Handoff Data Call Blocking Probability

\section{Acknowledgements}

I would like to acknowledge the advice and wise counsel of the co-authors Dr. Roseline N. Akol and Dr. Julius Butime who also supervised me for my Master of Engineering research; their contribution was vital in production of this manuscript. I would also like to recognize Mr. Alex Wanda who offered me the MATLAB program software free of charge. The program was much useful in production of the simulations as presented in section 3 .

\section{References}

[1] M. Al-Sanabani, S. Subramaniam, M. Othman \& Z. Zukarnain, "Mobility prediction based resource reservation for handoff in multimedia wireless cellular networks", The International Arab Journal of Information Technology, Vol.5, No.2, pp.162-169, April 2008

[2] Y. Li, C. Tian, S. Diggavi, M. Chiang \& A. R. Calderbank, "Network resource allocation for competing multiple description transmissions", IEEE Transactions on Communications, vol. 57, no. 5, pp. 1390-1401, May 2009

[3] P. Jain \& S. C. Gupta, "Hybrid mixed handover for call blocking probability analysis in wireless ATM network", Journal of Engineering and Computer Innovations, Vol. 2(4), pp. 68-77, April 2011

[4] K. Kamaluddin, "Dynamic channel allocation in mobile wireless cellular networks in heavy load conditions- Time Slot Division method", International Conference on Communication, Computer and Power (Icccp'09) Muscat, pp. 410-412, February 15-18, 2009.

[5] K. Sachan, R .C. Jain \& J. L. Rana "Dynamic channel allocation in cellular mobile communication", Adit Journal of Engineering, Vol. 2, No.1, pp. 3-6, December 2005

[6] Y. Fang \& Y. Zhang, "Call admission control schemes and performance analysis in wireless mobile networks", IEEE Transactions on Vehicular Technology, Vol. 51, No. 2, pp. 371-382, March 2002.

[7] T. S. Randhawa \& R.H.S. Hardy, "Performance analysis of multi-service cellular networks with mobile users", IEEE Wireless Communications and Networking Conference, Vol. 3, pp. 1390 - 1397, September 2000.

[8] C. M. Grinstead \& J. L. Snell, "Introduction to Probability", 2nd Ed., American Mathematical Society, 1997, Theorem 11.2, Chapter 11, pp. 409, Retrieved from: http://www.dartmouth.edu/ chance/teaching_aids/books_articles/probability_book/amsbook.mac.pdf

[9] T-C. Chan, K.Y. M. Wong \& B. Li "'Optimizing call admission control with QoS guarantee in voice/data integrated cellular network using anealing”, pp. 810 - 811, IEEE Globecom, Vol. 1, pp. 908-813, November 2002.

[10] A. Elnaggar, M. Aboelaze \& M. Musleh “A call admission control protocol for multimedia cellular networks”, Journal of Networks, Vol. 3, No. 8, pp. 45-54, November 2008

[11] Ojesanmi O.A, Oyebisi T.O., Oyebode E.O. \& Makinde O.E., "Performance analysis of congestion control scheme for mobile communication network", International Journal of Computer Science and Telecommunications, Vol. 2, Issue 8, pp. 33-36, November 2011. 